\title{
La propuesta inmanentista de Amartya Sen para la justicia global ${ }^{1}$
}

\author{
Amartya Sen's immanentist approach \\ to global justice \\ J. Francisco Álvarez \\ UNED \\ jalvarez@fsof.uned.es
}

Resumen. En The Idea of Justice (TIJ) de Amartya Sen se presenta una propuesta para comprender y defender la justicia global, alejada de las teorías de la justicia que se apoyan en el contrato social y en nociones institucionales trascendentales. El libro puede considerarse como un intento sistemático de defender la pertinencia de la tradición de la elección social (desde Condorcet a Arrow) para una noción robusta de justicia que no requiera una visión monolítica ni una única formulación institucional. Nos propone atender a las injusticias patentes, aunque no tengamos una teoría de la justicia e incluso aunque no compartamos elementos centrales para esa teoría de la justicia. Es un auténtico desafío a la posición estándar en filosofía moral.

Palabras clave: justicia global, capacidades, objetividad y posición, injusticia, información, Rawls, Adam Smith, Condorcet, contrato social, institucionalismo trascendental, Niti, Nyaya, democracia y argumentación.

El desplazamiento analítico que nos plantea Amartya K. Sen en The Idea of Justice (TIJ) consiste básicamente en recomendar que atendamos principalmente a las situaciones de injusticia que se puedan remediar, en vez de pretender centrar nuestra mirada en la búsqueda más o me-
Abstract. The Idea of Justice (TIJ) by Amartya Sen appears as a proposal both to understand and defend global justice far from theories of justice supported on social contracts and transcendental institutional notions. The book can be regarded as a systematic attempt to show us how pertinent traditional social choice theory (from Condorcet to Arrow) can be in order to develop a strong notion of justice that does not require neither a monolithic perspective nor a single institutional formulation. A. Sen propounds us to take care of patent injustices though we do not have a theory of justice, and even if we do not share central elements to this theory of justice. It is a real challenge for standard position in moral philosophy.

Key words: global justice, capability, positional objectivity, injustice, information, Rawls, Adam Smith, Condorcet, impartiality, social contract, trascendental institutionalism, Niti, Nyaya, democracy by discussion.

nos definitiva sobre aquello que sea o pueda ser la naturaleza última de la justicia perfecta. Desde luego que dicha sugerencia tiene que ver, sin duda, con una clara y definida intencionalidad de intervención crítica en nuestro mundo social que trate de avanzar y formular aquellas 
situaciones que mejoren las condiciones de vida de las personas concretas.

Sin embargo, no es que en $T I J$ se defienda una ingenua propuesta que pretenda reducir el objetivo de la filosofía moral al ejercicio práctico de alguna suerte de organización asistencial. Antes al contrario, aunque el diagnóstico de las situaciones injustas y nuestro sentido de injusticia sea para Sen un punto de partida indispensable, no nos está proponiendo reducir nuestro papel a las cuestiones prácticas del presente para intentar ponerles remedio, sino que es a partir precisamente de nuestra indignación moral y de nuestro rechazo a situaciones injustas como se produce la reflexión crítica, la argumentación y la investigación que nos permite llegar a comprender la naturaleza de conceptos políticos y éticos como el de justicia o injusticia.

Así ocurre que asuntos como el de la objetividad de nuestras estimaciones de las situaciones injustas y de los remedios que podríamos proponer, el esfuerzo por conseguir la imparcialidad de nuestras propuestas más allá de nuestros intereses personales, o de nuestros prejuicios o presupuestos derivados de nuestro entorno más cercano, se convierten en temas clave para una idea de justicia suficientemente crítica, razonada y operativa.

Tomarnos en serio ese punto de partida concreto del sentimiento de injusticia se convierte así en la herramienta conceptual principal para una adecuada reflexión sobre los problemas de la justicia y de la justicia global, para presentar «una teoría de la justicia en sentido amplio» (p. ix). La tarea no es el dar respuesta a cuál sea la naturaleza última de la justicia perfecta, sino más bien cómo podemos abordar en la práctica la cuestión de ampliar la justicia y reducir la injusticia. El supuesto de que el ejercicio comparativo no se puede realizar sin identificar primero las demandas de la justicia perfecta se somete a una potente revisión crítica en el capítulo cuatro de TIJ, «Voz y elección social» (pp. 87113), donde se presentan de hecho los problemas y procesos que quedarían excluidos en un enfoque realizado a partir de los «principios rawlsianos de justicia», tras haberlos presentado y analizado previamente en una de sus mejores y más fuertes versiones. En partícular hay seis grandes temas que se presentan como exclusiones problemáticas y que van a tratar de incorporar a lo largo de todo el libro apoyándose fundamentalmente en una versión muy precisa de la teoría de la elección social. Esas exclusiones serían los temas comparativos de justicia al centrarse exclusivamente en la identificación de una sociedad justa perfecta, el atender exclusivamente a las instituciones justas olvidando una perspectiva más amplia que incluya las realizaciones sociales, el obviar los efectos posiblemente perversos de las actividades realizadas en un país sobre las personas que viven más allá de sus fronteras y el silencio de sus voces, el no disponer de mecanismos sistemáticos para corregir la influencia de los valores locales que pueden resultar nocivos cuando una sociedad se mantiene separada del resto, el no permitir que al menos en la posición original personas diferentes puedan mantener - después incluso de una intensa y larga discusión pública - principios diferentes sobre la justicia debido a sus normas y valores políticos razonados - no por diferencias en intereses personales-, y, por último, porque no dejan espacio a que algunas personas no siempre se comporten «razonablemente» a pesar del contrato social hipotético y ello pueda afectar a la adecuación de las instituciones propuestas. Sen reconoce que el último de los problemas ha sido analizado bajo la rúbrica de la distinción entre teorías «ideales» y «no-ideales», pero que todos los problemas anteriores no se resuelven simplemente diciendo que una cosa es la teoría 
ideal y otra la formulación práctica sino que en su conjunto muestran la inadecuación (también teórica) de las concepciones trascendental institucionalistas como la de John Rawls.

Puede decirse que con TIJ estamos ante una obra de síntesis, de recopilación de posiciones y enfoques, pero cuyo conjunto produce la emergencia de una concepción general sobre el amplio ámbito de la justicia, de sus relaciones con la vida individual, con la política, con las prácticas sociales. Además recoge grandes tradiciones del pensamiento político y social que terminaron concentrándose en la segunda mitad del siglo XX en la teoría de la elección social y en el aprovechamiento de importantes resultados de la teoría de la elección racional.

Aunque Sen trata de presentar un balance muy cuidadoso, y profundamente respetuoso, de los autores que han desarrollado en el siglo Xx la teoría de la justicia, señeramente la obra de Rawls, a quien le dedica largos y elogiosos comentarios, sin embargo en TIJ se produce desde el mismo inicio una separación radical respecto de buena parte de esas concepciones que van a ser calificadas de trascendentalistas. No se trata de preguntar cuáles serían las instituciones perfectamente justas sino más bien cómo podríamos expandir la justicia y reducir la injusticia. Amartya Sen propone una doble ruptura con los enfoques del institucionalismo trascendental que siguen más o menos la línea del «contrato social» (Hobbes, Locke, Rousseau y Kant), defiende la oportunidad de un camino comparativo en oposición al trascendental y se propone fijar la atención en las prácticas y en los resultados concretos obtenidos en las sociedades analizadas en vez de atender exclusivamente a las normas e instituciones que se dan en ellas.

Así, el institucionalismo y el trascendentalismo, que pueden darse unidos en las propuestas del contrato social, aparecen como dos focos a evitar porque desde ellos no parece posible comprender la necesidad de acciones dirigidas a corregir, mejorar y tratar de eliminar las claras situaciones de injusticia en un plano global. Precisamente ese diferente punto de partida es el que le coloca en línea con «otra» tradición (p. 8), con una segunda línea ilustrada, de más o menos el mismo período (Adam Smith, Condorcet, Wollstonecraft, Bentham, Marx, Stuart Mill) y le permite avanzar desde el comienzo hacia una formulación de la idea de justicia global. La imposibilidad teórica de hablar coherentemente de la justicia global por parte de autores como Rawls o Thomas Nagel (a quien también dedicaba párrafos muy elogiosos de tenor parecido a los dedicados a Rawls) se destaca desde el comienzo del libro como uno de los desafíos que pretende afrontar y resolver. La propuesta de Amartya Sen aparece como una formulación coherente de una propuesta práctica para avanzar activamente en los procesos de incremento de la justicia global a partir de una teoría plural, centrada en los mecanismos de realización y en los resultados, que articule los elementos vertebradores de la idea de justicia global.

Situar la importancia de las instituciones con su destacado papel instrumental para perseguir la justicia no significa que tengamos que definir los principios de la justicia en términos de las instituciones, por lo que Sen propone formularlos más bien en términos de las vidas y libertades de las personas. Ni hay que presuponer la existencia de un único modelo institucional ni tampoco la pertinencia de unos principios únicos de justicia a partir de los cuales deba establecerse el acuerdo; por el contrario, al adoptar una posición pluralista radical que no considera a la democracia exclusivamente como el establecimiento de instituciones específi- 
cas, sino como un procedimiento que abre cauces a la argumentación pública, de hecho se permite y se facilita el avance de la democracia y la justicia global de manera que se puede lograr influir en las prácticas que se realicen en muy diversas partes del mundo.

El debate público, no lejano a la idea milliana del gobierno por discusión, es la línea articuladora de un modelo democrático fruto de muy diversas corrientes intelectuales y culturales, no exclusivamente «occidentales», que abraza Sen en toda la obra, en clara continuidad con lo que ha venido planteando durante muchos años y que ya había desarrollado con detalle en su obra The Argumentative Indian (2005). Nos plantea una noción de democracia que va más allá de las instituciones que existen formalmente y que se propone atender a la medida en que de hecho se expresan y se oyen diferentes voces surgidas de diversas partes del pueblo. Desde esa noción argumentativa y discursiva aparece la democracia global y la justicia global como ideas plenamente comprensibles que pueden inspirar e influir en determinadas acciones prácticas más allá de las respectivas fronteras (p. xiii).

Al superar la visión trascendental de las instituciones justas se establecen las condiciones iniciales para poder hablar sobre, y proponer mecanismos de justicia global; principalmente entendidos como mecanismos de superación de las injusticias. No hace falta «esperar» al buen día del acuerdo universal sobre algún tipo de contrato universal (antes pensado trascendentalmente para el Estado-nación) ni necesitamos disponer de una teoría general de la justicia que nos permita un orden completo de preferencias sobre los distintos estados posibles. Esa situación de exigencia inicial es la que convierte en vacío lo que puedan decir los trascendentalistas porque «La justicia global perfecta realizada por medio de un conjunto de instituciones impecablemente justas, incluso en el caso de que pudieran identificarse, ciertamente demandarían un Estado global soberano, y en la ausencia de un estado tal, los asuntos de la justicia global aparecerán ante los trascendentalistas como totalmente inmanejables») (p. 25). Así, por ejemplo, analiza con todo detalle cómo Thomas Nagel, a quien por otra parte reconoce como amigo y colega del que ha aprendido mucho, a partir de una concepción trascendental de la justicia no puede sino concluir que la justicia global no es un tema viable, porque las demandas que Nagel considera necesarias para un mundo justo no pueden alcanzarse a nivel global en estos momentos.

En cierta manera Sen trata de extender sus trabajos en teoría de la elección social para mostrar cómo hay muchos aspectos de dicha teoría que resultan sumamente significativos para una teoría de la justicia, aunque «las principales teorías filosóficas sobre la justicia le parezcan a muchos más cercanas al mundo de la práctica que lo que puede pretender la teoría de la elección» (p. 95). De hecho todo el libro puede considerarse como un intento sistemático de defender la pertinencia de la tradición de la elección social (desde Condorcet a Arrow), para poder avanzar en una idea de la justicia que permita disponer de una noción robusta de justicia a la par que no requiera una visión monolítica ni una única formulación institucional. En particular todo el trabajo comparativo que puede realizarse para ordenar los diversos resultados desde un punto de vista social, a partir de las estimaciones realizadas por las personas, nos lleva a terrenos muy diferentes de los que conducirían a la búsqueda de la «alternativa suprema entre todas las posibles», que es en lo que se han centrado las teorías de la justicia de Hobbes a Rawls. En términos de Sen: 
La distinción es importante... Un enfoque trascendental no puede, por sí mismo, manejar las cuestiones relativas al avance de la justicia y comparar las propuestas alternativas para conseguir una sociedad más justa, más allá de la propuesta utópica de imaginarse algún salto hacia un mundo perfectamente justo. Efectivamente, las respuestas que un enfoque trascendental da o puede dar a la justicia son muy diferentes y distantes del tipo de cuestiones y preocupaciones que tienen las personas interesadas en discutir sobre las cuestiones de justicia e injusticia en el mundo (por ejemplo, los males del hambre, la pobreza, el analfabetismo, la tortura, el racismo, la esclavitud de las mujeres, el encarcelamiento arbitrario o la exclusión de la atención sanitaria como asuntos sociales que precisan remediarse) (p. 96).

Aunque no aparecen en este libro asuntos muy nuevos respecto a las propuestas que ha venido avanzando Sen durante los últimos decenios en múltiples artículos, libros y contribuciones, tampoco es que aquí simplemente se reúnan materiales que se encontraban dispersos. Al organizar esas contribuciones el libro ha tenido ya un importante impacto porque, por un lado, ofrece una cierta bitácora que orienta y articula muchas de sus aportaciones en los últimos cuarenta años $\mathrm{y}$, principalmente, porque realiza esa tarea atendiendo centralmente a la idea de justicia. Además, si añadimos la reciente aparición de un libro colectivo Against Injustice: The New Economics of Amartya Sen (2009), con el artículo de apertura y la respuesta a los críticos a cargo de Sen, me parece que TIJ constituye una aportación clave para los temas contemporáneos de la teoría de la justicia. Se muestra allí con claridad cómo el enfoque de Sen es una separación muy notable de la tradición en teoría de la justicia, pues se trata de pensar en las injusticias, en las injusticias patentes, aunque no tengamos una teoría de la justicia e incluso aunque no compartamos elementos centrales para esa teoría de la justicia. Es un auténtico desafío a la posición estándar en filosofía moral.

Esa revisión crítica profunda de la concepción recibida en filosofía moral aparecía ya claramente formulada en su trabajo de 2002 («Open and Closed Impartiality», The Journal of Philosophy, 99, 9: 445-469), en el que insistía en dos nociones bien diferentes de imparcialidad: aquélla que atiende a una imparcialidad «interna» referida al grupo focal que establece un «contrato social» entre los miembros de un determinado cuerpo político y, otra bien diferente, que se preocupa por superar la visión local, la perspectiva del propio grupo y demanda algún tipo de observador imparcial externo en la línea de la propuesta lanzada en su momento por Adam Smith.

Frente a la propuesta de una teoría general sobre la justicia, Sen se dispone a reflexionar sobre la idea de justicia para encontrar los rasgos distintivos y la posibilidad de establecer una discusión, un debate racional sobre aquellos rasgos que pueden caracterizar la misma idea de justicia. En esta nota, más que entrar a sintetizar toda la riqueza conceptual y de matizaciones que se hacen en TIJ, indicaré algunos aspectos metodológicos que puedan ayudar a la muy recomendable lectura atenta del libro.

Para esa tarea me parece muy conveniente sobrevolar primero un poco sobre lo que se nos dice en este libro para inmediatamente pasar a recordar algunos aspectos claves de la historia de las intervenciones de Sen sobre muchos de estos temas a lo largo de los últimos cuarenta años. Como ha señalado recientemente Chris Brown en su interesante reseña «On Amartya Sen and The Idea of Justice» (Ethics \& International Affairs, 24, n. ${ }^{\circ} 3,2010$, pp. 309-318), la obra «resume y amplía muchos de los temas más importantes sobre los que ha trabajado Sen durante el último cuarto de siglo». 
Podríamos comenzar por recordar el importante seminario o curso conjunto ofrecido en Harvard a finales de los años 60 del pasado siglo y que reunió a tres pensadores claves de la segunda mitad del siglo xx: John Rawls, Kennett Arrow y Amartya Sen. Como ha recordado Sen en su nota autobiográfica para la Fundación Nobel, ese curso conjunto sobre «justicia social» ayudó mucho a la versión final de su Collective Choice and Social Welfare (1970). El curso tuvo un éxito notable y «terminó siendo bien conocido fuera del campus. En un viaje de avión a San Francisco mi vecino de asiento me preguntó si como profesor en Harvard había oído hablar de un curso "aparentemente muy interesante" ofrecido, por Kennett Arrow, John Rawls y un joven profesor desconocido». Los contrastes y las diferencias entre ellos, sus acuerdos respecto al espacio de investigación han resultado claves para la teoría política, para la metodología de la ciencia económica e incluso para el establecimiento de campos de investigación como la teoría de la elección social y la redefinición de la elección racional. Ya en la primera edición de $A$ Theory of Justice de Rawls (1971) se reconoce que sus fundamentos en teoría de la elección se sustentaban en la obra de Sen.

Quizás por esa línea podríamos entender mejor lo particularmente pertinente que resulta $T I J$ para un tema como el que se aborda en este número de Isegoría con el énfasis en la justicia global. Sin duda uno de los puntos calientes en este asunto es también el aspecto teórico, cómo es posible la formulación de una teoría general sobre la justicia global, cuáles son sus fuentes de legitimidad, cuáles son sus condiciones de posibilidad, cómo podemos hacer que tenga significatividad social y política.

Esos problemas aparecen en el núcleo mismo de este libro de Sen y mues- tran la pretendida (creo que con razón) superioridad de su propuesta sobre la de Rawls por lo que respecta a la posibilidad de abordar el tema de la justicia global. Estamos, sin duda, ante una contribución de plena madurez, en la que se produce una cierta recapitulación de su obra anterior y se muestra uno de los elementos centrales de toda su producción: la preocupación por decir algo práctico sobre los temas del presente. Sus trabajos sobre el hambre, la pobreza extrema, la situación de las mujeres, los problemas derivados de una visión monolítica de las identidades e incluso los problemas del terrorismo global, aparecen con toda legitimidad en esta obra en la que se insiste en primer lugar que se trata de ofrecer una salida ante una vía que resultó fallida en el camino hacia una teoría general de la justicia. Como dijo Kofi A. Annan, quien era Secretario General de las Naciones Unidas cuando Amartya Sen fue laureado con el Nobel en Economía (1998): «Los pobres y desposeídos del mundo puede que no tengan un defensor más penetrante y sistemático entre los economistas que Amartya Sen. Al demostrar que la calidad de nuestras vidas no se debería medir por nuestra riqueza, sino por nuestra libertad, sus escritos han revolucionado la teoría y la práctica del desarrollo. Naciones Unidas, en su propio trabajo sobre el desarrollo, se ha beneficiado inmensamente de la sabiduría y el buen sentido de los puntos de vista del profesor Sen».

Ahora bien, como en otra ocasión he planteado (Álvarez, 2001, «Capacidades, libertades y desarrollo: Amartya Kumar Sen», en Máiz, R., comp., Teorías políticas contemporáneas), la evidente disposición de Sen a extraer las consecuencias políticas de su elaboración teórica, le ha llevado a no restringir la incidencia de sus estudios, incluso los referidos a la pobreza y el hambre, como muchos quizás 
pretenderían, al exclusivo entorno de los países «pobres» y ponía como ejemplo su reflexión crítica sobre la moderación presupuestaria o conservadurismo financiero. Si por conservadurismo financiero se entiende la tendencia a tener un presupuesto equilibrado nada habría que objetar, pero Sen critica con fundamentación y detalle la pretensión de discutir sobre el déficit teniendo en cuenta exclusivamente los gastos referidos a la seguridad social, a la atención al desempleo o a otros servicios sociales públicos, sin colocar en el mismo plano de la discusión pública otros gastos como los militares, los de seguridad o la reducción de los gastos en $\mathrm{I}+\mathrm{D}$ debido a la fuerte inversión en investigación militar.

En el fondo se trata de una defensa de la acción pública, la discusión y la participación democrática como pieza clave para diseñar políticas económicas que atiendan a los más desfavorecidos y que se preocupen por la igualdad. Precisamente por ello Sen ha venido planteando, por ejemplo en Development as Freedom (1999), que el desarrollo múltiple de las libertades y su ejercicio democrático es una condición básica para la eficacia de programas auténticamente igualitarios. Incluso ha planteado con total claridad que su trabajo trata de ofrecer respuestas a cuestiones que se proponía resolver el socialismo y la izquierda clásica, que siguen abiertas y siguen siendo urgentes, aunque las lecciones de las diversas experiencias históricas nos indiquen que las formas prácticas de resolución no hayan sido precisamente eficaces o que no hayan tenido suficientemente en cuenta la justicia de los procesos de transición.

En este enfoque se destaca la importancia de conocer bien los efectos de las diversas políticas, los «costes» de cada una de ellas y la relevancia de los procedimientos que se aplican. Se trata de una perspectiva «comprensiva» que está atenta al procedimiento y a los resultados. Por ello aparece como fundamental la reflexión sobre los medios para no quedar prisioneros de viejas concepciones que asignan, sin auténtica comprobación práctica, determinadas soluciones (por ejemplo, la propiedad pública) al pensamiento de izquierda igualitarista. Comprender mejor los procesos de asignación que se producen mediante mecanismos democráticos puede ser una de las vías para articular mejor el binomio igualdad y libertad.

Las vías contractualistas y las transcendentalistas se muestran como inadecuadas para los problemas que debería abordar la filosofía moral y la filosofía política en el presente, la idea de justicia necesita de otros mimbres bien diferentes y trata de buscar entre la práctica social nuestros referentes para comprender y estimar una determinada situación como injusta. En línea con lo que alguna vez escribió John Roemer en A general Theory of Exploitation and Class (1982), se trata de avanzar en la importancia de una sociología de la injusticia más allá de la teoría de la justicia.

Hay dos o tres elementos conceptuales casi imprescindibles para situar adecuadamente la reflexión sobre la justicia que aparece en TIJ en el conjunto de la obra de Sen. En particular, en primer lugar, nos encontramos con el amplio tema de la información disponible para el individuo y en una determinada situación social, en segundo lugar, es preciso comprender el juego entre objetividad y pluralidad $y$, en tercer lugar, resulta fundamental distinguir adecuadamente entre unos pretendidos caminos ideales hacia los óptimos sociales y los auténticos caminos prácticos que tratan de satisfacer de la mejor manera nuestros objetivos de justicia.

Al tratar de precisar esos rasgos metodológicos que facilitan la comprensión 
de la obra de Sen, se comprenderán bien los elogios que, a lo largo de todo el libro y en múltiples ocasiones, se dirigen a la obra de Rawls y, a la vez, la profunda y radical crítica que se hace de la perspectiva rawlsiana por su enfoque trascendental contractualista y por no poder acoger la pluralidad como fuente de actividad, debate y reflexión, en cuyo seno cabe actuar para superar las injusticias.

La conocida formulación de Sen de la imposibilidad de un liberal paretiano, por la que se muestra la inadecuación del Criterio de Pareto con unos mínimos de derechos individuales reconocidos, y su revisión del teorema de imposibilidad de Arrow, que formulaba la inviabilidad de la existencia de una función de bienestar social que respetase simultáneamente el principio de no dictadura y un mínimo de requerimientos razonables sobre las preferencias de los individuos, son dos piezas centrales a la hora de destacar el importante papel de la información disponible a la hora de formular estimaciones sobre la justicia de una determinada situación social. Esa consideración de la información disponible como elemento básico, lo que de paso apunta a su destacada atención a la argumentación y al debate público, aparecía ya en sus formulaciones de la noción de bienestar como felicidad, en sus análisis sobre el nivel de vida y sobre las diversas formas de consideración de la idea de igualdad, "¿igualdad de qué?», analizada en su Inequality Reexamined (1995). Un camino que a partir de su reflexión sobre el nivel de vida (The Standard of Living, 1988), pasando por la elaboración de índices complejos para mostrar la combinación del nivel absoluto y el nivel relativo de pobreza, le condujo al desarrollo de sus teorías sobre el entitlement, clave para comprender su enfoque absolutamente innovador sobre el problema de las hambrunas al mostrar que no se trataba de un problema de carencia de disponibilidad de alimentos sino de ausencia de derechos de los individuos a demandar los alimentos existentes, y llegar finalmente al enfoque de las capabilities, es todo un proceso continuado de esfuerzo para mostrar la pertinencia de la información disponible por parte de los agentes individuales; más allá del rational fool que no tiene en cuenta la importancia de los elementos sociales, las normas de conducta, incluso la etiqueta, a la hora de organizar la información y los elementos de selección de la información por parte de los agentes. La información y el acceso a ella aparecen como fuentes claves de la conducta social. Sen ha insistido en muy diversos momentos en la idea de que nuestros juicios morales actúan como filtros informativos que dejan pasar cierta información e impiden que sean tenidas en cuenta otras informaciones importantes. Una de esas informaciones es la referida al individuo y a sus libertades, que deberían incluir no solamente los bienes de los que el individuo dispone, sino su misma capacidad de transformación de esos bienes en libertades concretas (aquí reside buena parte de la crítica de Sen a Rawls, con independencia de que comparta algunas de las motivaciones básicas que están detrás de entender la justicia como equidad). El peculiar enfoque de Sen sobre las relaciones entre ética y economía, en cuyas interrelaciones se encuentra su forma de obtener una idea de justicia, puede entenderse a partir de su propuesta metaética de considerar a los principios morales desde el punto de vista de la información. Como él mismo ha dicho:

El fundamento o apoyo informativo de un juicio identifica la información de la que depende directamente el juicio y - no menos importante - afirma que la verdad o falsedad de cualquier otro tipo de información no puede influir directamente en la corrección de tal juicio. La base informativa de nuestras consi- 
deraciones o juicios de justicia determina así el territorio fáctico sobre el cual son directamente aplicables esas consideraciones de justicia. (Las implicaciones sobre otras materias deben ser derivadas) (A. Sen, «Justice: Means versus Freedoms», Philosophy and Public Affairs, 11, 1990, p. 111).

Queda así clara la importancia asignada por Sen a la base informativa. Otro aspecto clave para comprender su formulación general es el peculiar enfoque sobre la pluralidad y la no optimización, que, de hecho, puede entenderse vinculado a la noción de racionalidad acotada de Herbert Simon como el mismo Sen ha indicado al mostrar cierta similitud de las nociones. Resulta particularmente pertinente analizar una noción de pluralidad que va más allá del simple reconocimiento de opciones dentro de una misma matriz conceptual. Es importante comprender que a veces cuando queremos ordenar no tenemos un criterio de conmensurabilidad perfectamente definido entre dos conceptos, y sin embargo podemos realizar entre ellos alguna comparación. Pero, sobre todo, es conveniente ver que más allá de un orden total, que tenga bien definido un primer elemento, o el elemento óptimo que domina toda la cadena de relaciones, podemos pensar en otras estructuras, lo que se conoce como orden parcial y la correspondiente noción de maximal y elementos maximales. El que puedan darse diversas dimensiones, la pluridimensionalidad, no significa que no se pueda hablar de nada, es más, en esos casos puede establecerse incluso un debate y una argumentación sistemática que diferencie y matice.

Sen aprovecha su excelente grado de experto en la teoría de la elección social para sugerirnos caminos que nos aclaren la senda de la justicia, particularmente que se opongan prácticamente a las injusticias manifiestas y, para ello, una herramienta interesante es mostrar la importancia de la dependencia del camino, del proceso, a la hora de proceder a la elección.

En esta misma línea aparece la preocupación y referencia constante por señalar que las contribuciones netas de las practicas sociales globales, la democracia, el constitucionalismo, la formulación de las libertades, la defensa de la crítica, no son fruto exclusivo de una supuesta civilización superior o cultura occidental que habría alcanzado su madurez en el mundo de la economía de mercado y en la organización democrática de la vida pública. Sen, tal y como ha venido planteando en múltiples momentos de su dilatada obra, insiste una y otra vez sobre los múltiples focos y las diversas contribuciones a la historia de la democracia. «El supuesto implícito de que la democracia pertenece exclusivamente a occidente, la aceptación de que es una idea esencialmente "occidental" que se ha originado y desarrollado solamente en occidente... es una idea difícilmente justificable... sin duda la estructura institucional de la práctica contemporánea de la democracia es en buena medida el producto de las experiencias europeas y norteamericanas de los últimos siglos, pero como Tocqueville recordaba a principios del XIX, la gran revolución democrática que se daba entonces en Europa y Norteamérica era una "nueva cosa" y era también la expresión de las más profundas, antiguas, permanentes y continuas tendencias conocidas en la historia» (pp. 322-323).

Sen hace un rápido repaso por contribuciones «no occidentales» de ese proceso, en particular las experiencias de India antigua. «No comprenderíamos las importantes demandas de una vida participativa, sobre lo que habló Aristóteles ampliamente y con intuiciones poderosas, si considerásemos que la democracia fuese un cierto tipo de producto cultural especial de occidente» (p. 322). Y todo esto tiene una gran importancia para la idea de 
justicia porque si las demandas de justicia, en el sentido de Sen, solamente pueden evaluarse con la ayuda del razonamiento público, y «si ese debate y razonamiento público está constitutivamente relacionado con la idea de democracia, aparece así una conexión íntima entre la justicia y la democracia, con rasgos discursivos compartidos» (p. 326).

Para mostrar algunos rasgos distintivos de la idea de justicia que trata de formular Sen resulta fundamental precisar la distinción entre proceso y resultado. En $T I J$ se hace un largo rodeo sobre dos nociones claves en el pensamiento de la India para abordar con suma precisión y diversos matices la distinción entre la moral de las consecuencias y la moral del proceso. Sen destaca la importancia del proceso al mismo tiempo que la relevancia de saber en cada momento quién es el agente que elige, decide y participa. De esta manera se destaca la importancia de lo que se ha llamado el empoderamiento de los agentes, la capacidad de ser sujeto agente con el potencial de intervenir en determinados procesos.

En TIJ introduce y explica una importante distinción que se da en la jurisprudencia india (y en la tradición cultural más amplia) entre dos nociones utilizadas para hablar de justicia: niti y nyaya. La primera hace referencia a las instituciones y reglas, a la norma legal, a las propiedades organizativas y a la adecuación de la conducta. Por otra parte, nyaya se enfoca sobre el mundo que emerge de las prácticas y no tanto a las normas o instituciones que tenemos, se utiliza para una concepto comprensivo de la justicia lograda. Sen desarrolla con mucho detalle esa distinción entre niti y nyaya, que resulta clave para todo su enfoque, comenzando por señalar que no se trata de la distinción entre un enfoque deontológico y uno consecuencialista. El punto de vista nyaya, atento a las realizaciones, a los resultados, no es un mero consecuencialismo que no tenga en cuenta el proceso exacto a través del cual llegamos a conseguir tal situación. Se trata de una consideración "comprensiva», incluyente, que tiene en cuenta la manera en la que se obtiene un resultado (por ejemplo, «un arresto arbitrario» no es la simple captura y detención de alguien), al mismo tiempo que incorpora el papel del ser humano como agente activo en el logro de esos estados. Estos han sido temas muy destacados en la obra económica de Amartya Sen, en TIJ hace referencia a su artículo de 1997, «Maximization and the act of choice», en el que se muestra la importancia de tener en cuenta el camino por el que se llega a conseguir un determinado resultado y que le servía allí para realizar una aguda crítica a la teoría de la elección racional por no incorporar ese tipo de dependencia al suponer que la elección se da en una suerte de vacío social e institucional.

Resulta particularmente interesante la vinculación que realiza Sen entre el enfoque nyaya y las posiciones de Adam Smith, Condorcet o Mary Wollstonecraft, quienes al defender el abolicionismo no pretendían decir que la abolición de la esclavitud iba a producir un mundo perfectamente justo: «su afirmación más bien era que una sociedad con esclavitud era totalmente injusta... La diagnosis de una injusticia intolerable en el caso de la esclavitud es lo que convirtió a la abolición en una prioridad insoslayable, y ello no requería la búsqueda de un consenso sobre cómo debería ser la sociedad perfectamente justa» (p. 21).

Quizás uno de los aspectos más importantes de esta contribución de Sen es que nos plantea que vale la pena trabajar sobre cosas del tipo ¿qué reformas internacionales es necesario hacer para que el mundo sea un poco menos injusto?, aunque pueda parecerles una pérdida de 
tiempo a los que están persuadidos (en clave hobbesiana o rawlsiana) de que necesitamos un Estado soberano para poder aplicar los principios de justicia mediante la elección de un conjunto perfecto de instituciones:

La teoría de la justicia como se formula en la corriente dominante del institucionalismo trascendental, reduce buena parte de los temas más pertinentes de la justicia a una retórica vacía - aunque sea significativa. Cuando las personas por todo el planeta se movilizan para exigir más justicia global $-\mathrm{y}$ destaco aquí el término comparativo «más»- no están demandando algún tipo de «humanitarismo mínimo». Tampoco se están moviendo impulsados por una sociedad mundial «perfectamente justa», sino que simplemente pretenden conseguir la eliminación de algunas situaciones escandalosamente injustas para ampliar así la justicia global... y también sobre qué acuerdos se pueden generar por medio de la discusión pública a pesar de que permanezcan las divergencias de puntos de vista en otros asuntos (p. 26).

Otras importantes piezas conceptuales aparecen en $T I J$ y sirven para apuntalar un importante proyecto crítico de las teorías contemporáneas de la justicia. Algunas de ellas surgen del propio desarrollo que la teoría económica ha tenido a manos de Sen. En particular, hay que destacar su enfoque de las capacidades potenciales (capability approach), que se relaciona estrechamente con la elección del foco informativo al que atendemos a la hora de juzgar una sociedad para evaluarla respecto a la justicia y a la injusticia. Partiendo de la idea de que cualquier teoría de la justicia tiene que adoptar ese tipo de decisión y frente a maneras de pensar que atienden a la utilidad individual (entendida como felicidad o placer, por ejemplo) u otras que atienden a los ingresos, recursos o riqueza (incluyendo los bienes primarios), Sen propone evaluar por la capacidad (la oportunidad real) que tienen las personas de hacer co- sas que tienen razones para valorar: «Se atiende aquí a la libertad que una persona efectivamente tiene para hacer esto o para ser aquello - asuntos que valora hacer o estima ser-》(p. 232). La noción de capacidades está estrechamente vinculada a la libertad considerada como oportunidad en términos de oportunidades globalmente consideradas y no fijándose exclusivamente en lo que resulta como culminación de un determinado proceso.

La cuestión central, insistirá Sen en discusión con Rawls, es que debemos distinguir entre libertad y el logro o éxito concreto. La «capacidad» refleja la libertad de una persona para elegir entre vidas alternativas, es decir, entre determinadas combinaciones de «funciones» que representan las cosas que podemos hacer y las diversas formas de ser. Al atender a la capacidad potencial es preciso considerar la transformación que cada uno realiza de los bienes primarios en logros concretos, pues hay condiciones diferentes entre los individuos. La capacidad representa la libertad, mientras que los bienes primarios nos hablan sólo de los medios para la libertad sin atender a la variación interpersonal entre esos medios y las libertades concretas conseguidas. Utilizando el resumen final que hace Sen en su artículo «Justice: means versus freedom»:

Somos diversos, pero lo somos de maneras diferentes. Un cierto tipo de variación se relaciona con las diferencias que hay entre nuestros fines y objetivos. Las implicaciones éticas y políticas de esta diversidad las entendemos ahora mejor que antes como resultado de los potentes trabajos de Rawls sobre la justicia como equidad. Pero hay otra diversidad importante: las variaciones en nuestra aptitud para convertir recursos en libertades concretas. Variaciones que hacen referencia al sexo, a la edad, a la dotación genética, y a muchos otros rasgos que nos dan potencia desigual para construir nuestra libertad en nuestras vidas, aunque tengamos la misma dotación de bienes primarios. 
Es importante atender a esos rasgos diferenciales, a esas diferencias entre los diversos individuos y las diversas comunidades, las diferentes instituciones y las formas diferentes de producir esas libertades concretas. Precisamente, esta atención a la diferencia puede venir a partir del estudio de los procesos políticos prácticos y mediante una atención a la política «real». Este aspecto de atender a las prácticas políticas reales es una de las constantes en las aportaciones de nuestro autor.

Con toda la importancia que tiene la noción de información no lo es menos la conformación social del propio individuo. Precisamente otra de las aportaciones metodológicas de Sen se refiere a cómo abordar este problema y constituye la que considero otra importante contribución metodológica: su análisis y reflexión sobre la noción de objetividad. La objetividad no aparece como el resultado de la ausencia de perspectiva, ni tampoco es resultado de suprimir todas las componentes individuales. Por el contrario, la objetividad inevitablemente es el resultado de una determinada perspectiva desde un preciso y específico lugar.

Sen ha sintetizado el problema utilizando para ello la noción de objetividad relativa a la posición (positional objectivity). La objetividad se considera que es la perspectiva que se adquiere desde algún determinado lugar (a view from somewhere), en oposición a la objetividad usualmente considerada como universal ausencia de perspectiva (a view from nowhere). Es decir, debemos tener en cuenta que el individuo no es eliminable, por mucho que, por ejemplo, en filosofía moral pretendamos máximas universalizables. Por el contrario, no debemos olvidar que siempre debemos mantener al individuo al menos como parámetro indispensable de la acción y en nuestros juicios pero, además y en muchos casos, al considerar a esos individuos como actores racionales nuestra conducta debe tener en cuenta la interacción intencional entre agentes intencionales. La formulación que hace Sen de esta idea de la objetividad relativa a la posición puede servir para comprender su articulado y complejo punto de vista sobre muchos problemas políticos de importancia.

Por ejemplo, sus importantes aportaciones sobre la situación y el papel de las mujeres en la sociedad, su defensa de la recuperación del espacio público para esa mitad de la humanidad, se enfrentan en muchas ocasiones a lo que aparecía como conocimiento establecido, en el mismo sentido en el que se suponía erróneamente que el hambre debía ser un problema de alimentos, así la superpoblación debería ser un problema de anticonceptivos o de los procedimientos para el control de la natalidad. Sin embargo, ocurre que se produce un mayor control simplemente con la incorporación de la mujer al sistema productivo y a los espacios políticos públicos. No siempre lo más aparente es lo más eficaz: las grandes hambrunas están más vinculadas al hecho de haberse roto la capacidad de demandar alimentos por parte de los vulnerables, su teoría del entitlement, y porque eso mismo provoca la rotura del sistema de reproducción social al fallar uno de los momentos del circuito productivo, no basta con dar alimentos a los que no lo tienen, siendo eso fundamental, sino facilitar la reproducción práctica de esos sistemas y su sostenibilidad dotando a las personas de su capacidad de demanda, de tener derecho a. Lo mismo ocurre en el caso de las mujeres. Sen ha estudiado con detalle, por ejemplo, el caso de China, en el cual, el establecimiento de criterios como «un único hijo» para controlar la natalidad provocaba la «desaparición», en la práctica, de muchas mujeres por vía del infanticidio de la primogénita, por ejemplo, o 
por las diversas formas sociales indirectas de incrementar la discriminación incluso en los elementos básicos de reproducción social, acceso a la alimentación o a la atención sanitaria.

Algunas de estas consideraciones metodológicas resultan particularmente pertinentes para comprender el lugar de $T I J$ en la obra de Sen y para penetrar con mayor profundidad en las líneas que lo articulan. Así el último capítulo de TIJ titulado «La justicia y el mundo» concluye con una importante observación que enlaza con mucha de la producción de Sen, con trabajos tan importantes para la teoría económica como su «Rational Fools» (1977) o «Maximization and the Act of Choice» (1996), en definitiva, se trata de considerar qué «tipo de criaturas somos los humanos», el problema de los modelos de seres humanos (en términos de Herbert Simon) y de su papel como agentes:

no quiero decir que los debates sobre las teorías de la justicia se puedan resolver retrotrayéndose a ciertos rasgos de la naturaleza humana, sino más bien indicar que diversas teorías de la justicia comparten algunos presupuestos comunes sobre cómo somos los seres humanos [...]. La evidente presencia de rasgos que nos llevan a sentir las dificultades de los demás, a sentir como propios la humillación y el dolor de otros, la preocupación por la libertad y nuestras capacidades para discutir, razonar, acordar y discrepar aunque no nos diga mucho sobre qué teoría de la justicia preferir sin embargo apunta a que la búsqueda de la justicia no es fácilmente eliminable de las sociedades humanas aunque se persiga de maneras muy diferentes (pp. 414-415).
Sen ha indicado en muchas ocasiones, incluso para su trabajo en los campos de la teoría económica, la necesidad de atender a un modelo de ser humano más allá del romo y plano planteado por la teoría económica, más allá del imbécil egoísta racional, y proponer una auténtica superación de la misma noción hobbesiana de la vida humana, corta, bruta, mala y solitaria. Más bien, la búsqueda de la comunicación entre las capacidades humanas básicas, capacidad de comprender, sentir con los otros y argumentar, hace que no estemos irremediablemente condenados a mantener vidas aisladas y sin comunicación (y no estamos condenados a que la única fuente de selección de información sea el mercado), antes al contrario: «Ya es muy malo que el mundo en el que vivimos tenga tantas carencias de uno u otro tipo (de seres hambrientos y personas bajo tiranías), pero sería aún más terrible si no fuésemos capaces de comunicarnos, replicar y argumentar».

Quizás para terminar podríamos decir que TIJ hace un gran esfuerzo porque los desfavorecidos y ofendidos del mundo puedan encontrar también su voz en el campo de las teorías de la justicia, de manera cercana a como Sen, por otros motivos, considera encontrar esa voz en el potente poema de Seamus Heaney con el que cierra su introducción (p. 27):

La historia dice: no hay esperanza a este lado de la tumba,

pero entonces, por una vez en la vida,

la tan anhelada ola de la justicia puede aparecer,

y la esperanza y la historia rimarán
1 Señalamos la importancia del libro de Amartya Sen, The Idea of Justice (Cambridge, Mass.: Belknap Press/Harvard U.P., 2009), para una reflexión y práctica de la justicia global. Aunque apareció relativamente pronto una traducción al español de Hernando Va- lencia con el título de La idea de la justicia (Madrid, Taurus, 2010), sin duda de gran utilidad para difundir la obra de Sen en nuestra lengua, no la hemos utilizado porque ya estaba escrito este texto cuando hemos accedido a ella. En http://www.lexisnexis.com/docu- 
ments/pdf/20080806034945_large.pdf se puede acceder libremente a una síntesis clara y muy explicativa del enfoque de Sen respecto a la justicia global, pues se recoge allí la presentación que hizo el autor ante el World Justice Forum celebrado en Viena del 2 al 5 de junio de 2008.
Este trabajo se ha realizado en el ámbito de dos proyectos de investigación financiados por el Ministerio de Ciencia e Innovación: «La trama de la argumentación: bases conceptuales e implicaciones filosóficas» (FFI2008-00085) y «Hacia una nueva fundamentación de la filosofía de las ciencias sociales» (FI2008-03607). 